April 1998. To appear in Bulletin of the London Mathematical Society.

\title{
NOETHERIAN CENTRALIZING HOPF ALGEBRA EXTENSIONS AND FINITE MORPHISMS OF QUANTUM GROUPS
}

\author{
EDWARD S. LETZTER
}

\begin{abstract}
We study finite centralizing extensions $A \subset H$ of Noetherian Hopf algebras. Our main results provide necessary and sufficient conditions for the fibers of the surjection spec $H \rightarrow \operatorname{spec} A$ to coincide with the $X$-orbits in spec $H$, where $X$ denotes the finite group of characters of $H$ that restrict to the counit of $A$. In particular, all of the fibers are $X$-orbits if and only if the fiber over the augmentation ideal of $A$ is an $X$-orbit. An application to the representation theory of quantum function algebras, at roots of unity, is presented.
\end{abstract}

\section{INTRODUCTION}

If $A \subset H$ is an extension of finitely generated commutative Hopf algebras (over an algebraically closed field $k$ ), then classical duality provides an exact sequence of affine algebraic groups,

$$
1 \rightarrow X \rightarrow \max H \rightarrow \max A \rightarrow 1
$$

where the multiplication of maximal ideals is obtained from the convolution of their associated linear characters (i.e., algebra homomorphisms onto $k$ ), and where $X$ consists of those linear characters of $H$ that restrict to the counit on $A$. However, supposing now that $A \subset H$ is a noncommutative extension of Hopf algebras, the preceding group structure does not - in most cases - apply to their primitive spectra. But $X$ can still be defined as before, and there are actions of $X$ on $H$ by automorphisms that fix $A$ pointwise (as explained in (2.1) below). Moreover, these automorphic actions on $H$ induce actions on its primitive spectrum, and these actions on the primitive spectrum reduce to the left and right multiplication by $X$ on $\max H$, under convolution, that appeared in the original commutative case (see (3.1)). One can therefore ask, in the noncommutative setting, whether the fibers of the correspondence from spec $H$ to spec $A$ are determined by the action of $X$, in a manner generalizing the classical theory. Our aim in this paper is to present an affirmative answer to this question, under hypotheses applicable to quantum function algebras at roots of unity.

Now assume that $G$ is a connected and simply connected complex semisimple Lie group, that $\ell$ is an odd positive integer prime to 3 if $G$ possesses a $G_{2}$ component, and that $\epsilon$ is a primitive $\ell$ th root of unity. Our main motivating example involves the Hopf algebra embedding $F_{0} \subset F_{\epsilon}$, as studied by De Concini, Lyubashenko, and Procesi $[7,8]$ (multiparameter analogs may be found in the work of Costantini and Varagnolo $[4,5]$ ). Here, $F_{0}$ is isomorphic to, and $F_{\epsilon}$ is a quantization of, the classical coordinate ring of $G$. Moreover,

1991 Mathematics Subject Classification. 16D30, 16S20, 16P40, 16W30, 81R50.

This research was supported in part by grants from the National Science Foundation.

Typeset by $\mathcal{A} \mathcal{M}$ S-TEX 
$F_{0}$ is contained within the center of $F_{\epsilon}$, and $F_{\epsilon}$ is a finitely generated projective $F_{0}$-module [7]. (Following the terminology of $[17,1.8]$, the embedding of $F_{0}$ into $F_{\epsilon}$ may be viewed as a covering of quantum groups.) Consequently, the group $X$ of characters of $F_{\epsilon}$ that restrict to the counit on $F_{\epsilon}$ is finite, and it follows from [8] that the fibers of the surjection prim $F_{\epsilon} \rightarrow \operatorname{prim} F_{0}$ are precisely the $X$-orbits in prim $F_{\epsilon}$, under certain addtional restrictions on $\ell$. As an application of our analysis below, the additional assumptions on $\ell$ can be removed; see (3.2i). (This last result also follows from recent independent work by Montgomery and Schneider [16] concerning Hopf Galois extensions.)

To briefly describe the primary context for our study, and our main theorem, let $H$ be a noetherian Hopf algebra containing an associative subalgebra $A$. Suppose further that $A$ is a right coideal of $H$ and that $H$ is a finite centralizing extension of $A$ (i.e., $H$ is generated as an $A$-module by finitely many elements $x$ such that $a x=x a$ for all $a \in A$ ). As before, let $X$ denote the (finite) set of characters of $H$ that restrict to the counit on $A$. In (2.9) we conclude that the following conditions are equivalent: (a) the primitive ideals of $H$ contracting to the augmentation ideal of $A$ all have codimension 1 in $H$, (b) the primitive ideals of $H$ contracting to the augmentation ideal of $A$ comprise a single $X$-orbit in $\operatorname{prim} H$, (c) the fibers of the surjection $\operatorname{prim} H \rightarrow \operatorname{prim} A$ are precisely the $X$-orbits in $\operatorname{prim} H,(\mathrm{~d})$ the fibers of the surjection spec $H \rightarrow \operatorname{spec} A$ are precisely the $X$-orbits in spec $H$.

The material in this paper was presented in the special session on Algebras, Algebra Cohomology, and Polynomial Identities, at the 102nd Annual Meeting of the American Mathematical Society, Orlando, January, 1996.

I am grateful to M. Costantini for the helpful comments used in (3.2).

\section{Noetherian Centralizing Hopf Algebra Extensions}

The main results of this section are found in (2.7) and (2.9).

2.1 Preliminaries. All of the algebras considered in this section are defined over a single base field $k$.

(i) The comultiplication and counit (augmentation map) of a bialgebra will always be denoted $\Delta$ and $\varepsilon$, respectively, and the antipode of a Hopf algebra will always be denoted $S$. We will use the notation $\Delta(b)=\sum b_{1} \otimes b_{2}$.

(ii) Let $B$ be a bialgebra, and let $\chi$ be a (linear) character of $B$ (i.e., $\chi$ is an algebra homomorphism of $B$ onto $k$ ). Let $k_{\chi}$ denote the one-dimensional $B$-module defined by the action $b .1=\chi(b)$. The assignment

$$
\sigma_{\chi}: b \mapsto \sum \chi\left(b_{1}\right) b_{2}, \quad \text { for } b \in B
$$

is an algebra endomorphism, which we will call, following [10], a (right) winding endomorphism of $B$. If $B$ is a Hopf algebra, then every winding endomorphism is an automorphism; see, e.g., $[10,1.3 .4]$. Furthermore, if $\chi^{\prime}$ is also a character of $B$, then $\sigma_{\chi} \circ \sigma_{\chi^{\prime}}=\sigma_{\chi^{\prime} * \chi}$, where $\chi^{\prime} * \chi$ is the convolution of these characters. Consequently, if $B$ is a Hopf algebra then the character group of $B$ acts on $B$ by automorphisms. 
(iii) Retaining the preceding notation, let $A$ be an associative subalgebra of $B$. Observe, when $\sigma_{\chi}$ acts as the identity on $A$, that $\left.\chi\right|_{A}=\left.\varepsilon\right|_{A}$, because

$$
\chi(a)=\chi\left(\sum a_{1} \varepsilon\left(a_{2}\right)\right)=\sum \chi\left(a_{1}\right) \varepsilon\left(a_{2}\right)=\varepsilon\left(\sum \chi\left(a_{1}\right) a_{2}\right),
$$

for all $a \in A$. Now assume that $A$ is a right coideal of $B$ (i.e., $\Delta(A) \subseteq A \otimes B$ ). In converse to the preceding, if $\chi$ acts as $\varepsilon$ on $A$ then $\sigma_{\chi}$ restricts to the identity on $A$. Also, the set $X$ of characters of $B$ restricting to $\varepsilon$ on $A$ is closed under convolution, and so forms of subgroup of the character group of $B$.

2.2. Let $H$ be a Hopf algebra, and let $V$ be an $H$-H-bimodule. Recall that the ad $H$ module structure on $V$ is defined by the left action

$$
\operatorname{ad}(h) v=\sum h_{1} \cdot v \cdot S\left(h_{2}\right),
$$

where $h \in H, v \in V$, and $\Delta(h)=\sum h_{1} \otimes h_{2}$.

Further recall, when $V$ has a one-dimensional ad $H$-submodule $k$-spanned by the element $n$, that

$$
\begin{aligned}
& n \cdot \sigma_{\chi}(h)=\sum n \cdot \chi\left(h_{1}\right) h_{2}=\sum\left(\operatorname{ad}\left(h_{1}\right) n\right) \cdot h_{2}= \\
& \qquad h_{1} \cdot n \cdot S\left(h_{2}\right) h_{3}=\sum h_{1} \cdot n \cdot \varepsilon\left(h_{2}\right)=\sum h_{1} \varepsilon\left(h_{2}\right) \cdot n=h \cdot n,
\end{aligned}
$$

for all $h \in H$, where $\chi$ is the character of $H$ defined by $\operatorname{ad}(h) n=\chi(h) n$.

2.3. We now review some elementary facts from noetherian ring theory (the reader is referred to [9] or [14] for further details). Let $R_{\alpha}$ and $R_{\beta}$ be prime noetherian rings, and let $M$ be an $R_{\alpha}-R_{\beta}$-bimodule that is finitely generated on each side.

(i) Suppose that $M$ is torsionfree (cf. $[14,3.4 .2]$ ) on each side; $M$ is then said to be a bond from $R_{\alpha}$ to $R_{\beta}$. Next, let $m$ be a nonzero normal element of $M$ (i.e., $R_{\alpha} \cdot m=m . R_{\beta}$ ), and let $a$ be an element of $R_{\alpha}$ for which $a \cdot m=0$. Then $0=a \cdot m=R_{\alpha} a \cdot m \cdot R_{\beta}=R_{\alpha} a R_{\alpha} \cdot m$, and so $a=0$. (Recall, from Goldie's Theorem, that every nonzero ideal in a noetherian prime ring contains a regular element.) We see, therefore, that the left and right annihilators of any nonzero normal element of $M$ are both equal to zero. If $R_{\gamma}$ is also a prime noetherian ring and $N$ is a bond from $R_{\beta}$ to $R_{\gamma}$, then there exists an $R_{\alpha}-R_{\gamma}$-bimodule factor of $M \otimes_{R_{\beta}} N$ that is a bond from $R_{\alpha}$ to $R_{\gamma}$; see, e.g., [20, 5.1].

(ii) Let $R$ be a noetherian ring, and let $N$ be an $R$-R-bimodule that is finitely generated on each side. Let $P_{\alpha}$ and $P_{\beta}$ be prime ideals of $R$, and suppose that $N$ is a bond from $R / P_{\alpha}$ to $R / P_{\beta}$. Assume further that there exists an automorphism $\sigma$ of $R$, and a nonzero element $n$ of $N$, such that $r . n=n . \sigma(n)$ for all $r \in R$. Observe that $0=P_{\alpha} \cdot n=n . \sigma\left(P_{\alpha}\right)$ and that $0=n \cdot P_{\beta}=\sigma^{-1}\left(P_{\beta}\right) . n$. Consequently, using (i), $\sigma\left(P_{\alpha}\right)=P_{\beta}$.

2.4. Let $H$ be a noetherian Hopf algebra containing a noetherian subalgebra $A$ such that $H$ is finitely generated as a left and right $A$-module. Assume further that every $H$-module composition factor of the finite dimensional left $H$-module $H \otimes_{A} k_{\varepsilon}$ is one dimensional, and let $X$ denote the set of characters of $H$ corresponding to these composition factors. 
Lemma. Let $P_{\alpha}$ and $P_{\beta}$ be prime ideals of $H$, and let $Q$ be a prime ideal of $A$.

(i) Suppose that there exists an $H$-A-bimodule factor of $H$ bonding $H / P_{\alpha}$ to $A / Q$ and an $A$-H-bimodule factor of $H$ bonding $A / Q$ to $H / P_{\beta}$. Then $\sigma_{\chi}\left(P_{\alpha}\right)=P_{\beta}$ for some $\chi \in X$.

(ii) Suppose that $P_{\alpha} \cap A$ and $P_{\beta} \cap A$ are both equal to $Q$. Then $\sigma_{\chi}\left(P_{\alpha}\right)=P_{\beta}$ for some $\chi \in X$.

Proof. (i) By (2.3i), there is a nonzero $H$ - $H$-bimodule factor $V$ of $H \otimes_{A} H$ that is a bond from $H / P_{\alpha}$ to $H / P_{\beta}$. Let $e$ denote the image in $V$ of $1 \otimes 1$. Observe that $e$ is nonzero since it is the generator of $V$ as an $H$-H-bimodule. Furthermore, since a.e =e.a for all $a \in A$, it follows that the ad $H$-module generated by $e$ is isomorphic, as a left $H$-module, to a nonzero factor of $H \otimes_{A} k_{\varepsilon}$. Consequently, $V$ contains an element $n$ such that $\operatorname{ad}(h) n=\chi(h) n$ for some $\chi \in X$. It now follows from (2.2) that $h . n=n . \sigma_{\chi}(h)$, for all $h \in H$, and it follows from (2.3ii) that $\sigma_{\chi}\left(P_{\alpha}\right)=P_{\beta}$.

(ii) It follows, for example, from $[11,1.1]$ that there exists an $H$-A-bimodule factor of $H / P_{\alpha}$ bonding $H / P_{\alpha}$ to $A / Q$. Similarly, there exists an $A$ - $H$-bimodule factor of $H / P_{\beta}$ bonding $A / Q$ to $H / P_{\beta}$. Part (ii) now follows from part (i).

2.5. (i) Let $R$ be an algebra. The set of prime ideals of $R$ will be denoted spec $R$ and the set of (left) primitive ideals of $R$ will be denoted $\operatorname{prim} R$; these sets will be endowed with the Jacobson (Zariski) topology. If $\mathcal{A}$ is a group acting on $R$ by automorphisms, one immediately obtains actions of $\mathcal{A}$ on $\operatorname{spec} R$ and $\operatorname{prim} R$.

(ii) Assume that $R$ is a finite centralizing extension of a subalgebra $U$ (i.e., assume that $R=U r_{0}+\cdots+U r_{s}$, for elements $r_{0}, \ldots, r_{s} \in R$, such that $u r_{i}=r_{i} u$ for all $0 \leq i \leq s$ and all $u \in U$ ). It is well known that $U$ is noetherian if and only if $R$ is noetherian (see, e.g., $[14,10.1 .11])$ and that if $P$ is a prime ideal of $U$ then $P \cap U$ is a prime ideal of $R$ (e.g., $[14,10.2 .4])$.

(iii) Assigning $P \cap U$ to each prime ideal $P$ of $R$ produces closed continuous surjections

$$
u: \operatorname{spec} R \rightarrow \operatorname{spec} U \quad \text { and } \quad u: \operatorname{prim} R \rightarrow \operatorname{prim} U
$$

with finite fibers. See, for example, [14, Chapter 10] for more details. (Finiteness of the fibers follows from $[19,3.4]$.)

(iv) Let $M$ be a simple left $U$-module, and let $r_{0}, \ldots, r_{s}$ be as in (ii). Then $R \otimes_{U} M=$ $r_{0} \otimes M+\cdots+r_{s} \otimes M$, and we see that $R \otimes U M$ is a semisimple $U$-module, of length no greater than $s$, with each $U$-module composition factor isomorphic to $M$.

(v) We will say that an $R$-module $V$ restricts to a character $\xi$ of $U$ if $u . v=\xi(u) v$ for all $u \in U$ and $v \in V$. Observe that any finitely generated $R$-module restricting to a character of $U$ must be finite dimensional. Furthermore, it follows from (iv) that a simple $R$-module $W$ restricts to $\xi$ if and only if $W$ is an $R$-module composition factor of $R \otimes_{U} k_{\xi}$. Consequently, there are only finitely many simple $R$-modules restricting to $\xi$, and so there are at most finitely many characters of $R$ restricting to $\xi$. (This last assertion also follows from (iii).)

2.6. Let $B$ be a bialgebra that is a finite centralizing extension of an associative subalgebra A. 
(i) In view of $(2.5 \mathrm{v})$, the set of characters of $B$ restricting to $\varepsilon$ on $A$ is finite.

(ii) The kernel of $\left.\varepsilon\right|_{A}$ will be denoted $A^{+}$. Note that $B A^{+}=A^{+} B$, that $B A^{+}$is contained within the augmentation ideal of $B$, and that $B / B A^{+}$is a finite dimensional algebra. Of course, if $A$ is a Hopf algebra, and if the inclusion of $A$ in $B$ is a homomorphism of Hopf algebras, then $B / B A^{+}$is the Hopf cokernel.

2.7. We now record our primary abstract result, stated in its most general form. A more condensed version is presented in (2.9).

Theorem. Let $H$ be a noetherian Hopf algebra that is a finite centralizing extension of an associative subalgebra $A$, and let $X$ denote the set of characters of $H$ that restrict to $\varepsilon$ on $A$. The following conditions are equivalent:

(i) Every irreducible $\mathrm{H} / \mathrm{HA}^{+}$-module is one dimensional.

(ii) Every irreducible $H$-module restricting to $\varepsilon$ on $A$ is one dimensional.

(iii) If $P_{\alpha}$ and $P_{\beta}$ are primitive ideals of $H$ for which $P_{\alpha} \cap A=P_{\beta} \cap A$, then there exists $\chi \in X$ such that $\sigma_{\chi}\left(P_{\alpha}\right)=P_{\beta}$.

(iv) If $P_{\alpha}$ and $P_{\beta}$ are prime ideals of $H$ for which $P_{\alpha} \cap A=P_{\beta} \cap A$, then there exists $\chi \in X$ such that $\sigma_{\chi}\left(P_{\alpha}\right)=P_{\beta}$.

Proof. (ii) $\Rightarrow$ (iv) Let $P_{\alpha}$ and $P_{\beta}$ be prime ideals of $H$ for which $P_{\alpha} \cap A=P_{\beta} \cap A$. It follows from $(2.5 \mathrm{v})$ and (ii) that every $H$-module composition factor of $H \otimes_{A} k_{\varepsilon}$ is one dimensional. Statement (iv) now follows from (2.4ii).

The remaining implications are immediate.

2.8. Retaining the notation of (2.7), assume further that $A$ is a right coideal of $H$. It follows from (2.1iii) and $(2.5 \mathrm{v})$ that $X$ is a finite subgroup of the character group of $H$, and that the set $\left\{\sigma_{\chi} \mid \chi \in X\right\}$ is precisely the group of winding automorphisms of $H$ that fix $A$ pointwise. Consequently, each fiber of the surjection $u: \operatorname{spec} H \rightarrow \operatorname{spec} A$ is a union of $X$-orbits.

The following is now an immediate corollary to (2.7), and presents a somewhat smoother alternative to that result.

2.9 Theorem. Let $H$ be a noetherian Hopf algebra that is a finite centralizing extension of an associative subalgebra $A$, and suppose that $A$ is a right coideal of $H$. Let $X$ be the finite group of characters of $H$ that restrict to the counit on $A$.

The following conditions are equivalent:

(i) Every irreducible $H$-module restricting to the counit on $A$ is one dimensional.

(ii) The primitive ideals of $H$ contracting to the augmentation ideal of $A$ comprise a single $X$-orbit in prim $H$.

(iii) The fibers of the surjection $u: \operatorname{prim} H \rightarrow \operatorname{prim} A$ are precisely the $X$-orbits in prim $H$.

(iv) The fibers of the surjection $u$ : $\operatorname{spec} H \rightarrow \operatorname{spec} A$ are precisely the $X$-orbits in spec $H$.

2.10 Remark. Retaining the assumptions of (2.9), but additionally supposing that $A$ is a Hopf subalgebra of $H$, it turns out that the choice of $\varepsilon$ in $(2.9 \mathrm{i})$ is somewhat arbitrary. To explain, let $\xi$ be any character of $A$ for which every irreducible $H$-module restricting to $\xi$ is one dimensional, and let $\chi$ denote one of the characters of $H$ restricting to $\xi$. Set 
$\sigma=\sigma_{\chi}$. Because $A$ is a Hopf subalgebra, $\sigma$ maps $A$ to itself automorphically. Now let $K$ be the kernel of $\xi$, and observe for $a \in K$ that $\varepsilon(\sigma(a))=\sum \chi\left(a_{1}\right) \varepsilon\left(a_{2}\right)=\chi(a)=\xi(a)=0$. Therefore, $\sigma(K)=A^{+}$, and so $\sigma(H K)=H A^{+}$. In particular, $\sigma$ induces an algebra isomorphism from $H / H K$ onto $H / H^{+}$, and so the irreducible $H$-modules restricting to $\varepsilon$ are also all one dimensional.

\section{Finite Morphisms of Quantum Groups}

In this part we outline some connections between the results in the previous section and the representation theory of quantum groups.

3.1. We first review the classical case (cf. [1, Chapter 4; 15, §9.3]), which was briefly sketched in the introduction. To begin, assume that $k$ is an algebraically closed field, and let

$$
1 \rightarrow X \rightarrow Y \stackrel{\pi}{\rightarrow} Z \rightarrow 1
$$

be a short exact sequence of $k$-affine algebraic groups. Recall that there is a corresponding embedding $A \subset H$ of finitely generated reduced commutative Hopf algebras, where $Z$ is the character group of $A$, and where $Y$ is the character group of $H$. Conversely, an epimorphism of algebraic groups can be associated to each embedding of finitely generated commutative $k$-Hopf algebras (that need not be assumed reduced - see, e.g., [15, 9.2.1112]).

Observe that $X$, viewed as a subgroup of $Y$, is equal to the group of characters of $H$ that act as $\varepsilon$ on $A$. Hence, by (2.1iii), $X$ acts on $H$ by winding automorphisms, and $\left\{\sigma_{\gamma} \mid \gamma \in X\right\}$ is precisely the set of winding automorphisms of $H$ that fix $A$ pointwise.

On the other hand, by imposing the group structures of $Y$ and $Z$ (i.e., convolution of characters) onto $\max H$ and $\max A$, respectively, we see that the subgroup $X$ of $Y$ acts on $\max H$ by left and right multiplication. Moreover, since $X$ is the kernel of $\pi$, it immediately follows that the induced multiplication actions on $\max A$ are trivial and that the fibers of the surjection $\max H \rightarrow \max A$ are exactly the $X$-orbits, under the right or left multiplication action, in $\max H$. Finally, it is easy to check that the $X$-orbits in max $H$ under the multiplication actions are precisely the $X$-orbits, in max $H$, induced by the winding automorphisms mentioned in the preceding paragraph.

In particular, for finite extensions of finitely generated commutative Hopf algebras over algebraically closed fields, the equivalent conditions in (2.9) may be deduced from the classical theory.

3.2. Now let $G$ be a connected, simply connected, complex semisimple Lie group. Fix an odd positive integer $\ell$ (prime to 3 if $G$ has a $G_{2}$ component), and let $\epsilon$ be a primitive $\ell$ th root of 1 .

(i) It is shown by De Concini and Lyubashenko that the complex quantum function algebra $F_{\epsilon}$ of $G$ (as specified in $[7, \S 9]$ ) contains a central sub-Hopf-algebra $F_{0}$ isomorphic to the ring of regular functions on $G$, and that $F_{\epsilon}$ is finitely generated as an $F_{0}$-module [7, $6.4,7.2]$. Furthermore, it follows from $[7,10.7]$ that every irreducible $F_{\epsilon}$-module restricting to the counit on $F_{0}$ is one dimensional. Hence, by $(2.9)$, the fibers of the surjections

$$
\operatorname{spec} F_{\epsilon} \rightarrow \operatorname{spec} F_{0} \quad \text { and } \quad \operatorname{prim} F_{\epsilon} \rightarrow \operatorname{prim} F_{0}
$$


are precisely the $X$-orbits in spec $F_{\epsilon}$ and prim $F_{\epsilon}$, respectively, where $X$ is the group of characters of $F_{\epsilon}$ restricting to the counit of $F_{0}$.

The preceding conclusion has been independently verified by S. Montgomery and H.J. Schneider, in their study of Hopf Galois extensions [16]. Also, under the additional assumption that $\ell$ is prime to the bad primes of the associated root system, the conclusion already follows from $[8, \S 4.10]$. The approach in $[7 ; 8]$ involves a detailed analysis of certain Azumaya algebras, obtained as localizations of factors of $F_{\epsilon}$, and precise calculations of the dimensions of the irreducible representations are obtained.

(ii) Assuming that $G$ is simple, an extension $F_{0} \subset F_{\epsilon}^{\varphi}$ of complex Hopf algebras is studied by Costantini and Varagnolo $[4 ; 5]$, where $F_{\epsilon}^{\varphi}$ is a multiparametric quantization of the coordinate algebra of $G$ (cf. $[6 ; 18])$ and $F_{0}$ is isomorphic to the ring of regular functions on $G$. It is shown in [4] that $F_{0}$ is contained within the center of $F_{\epsilon}^{\varphi}$ and that $F_{\epsilon}^{\varphi}$ is finitely generated as an $F_{0}$-module. Furthermore, it follows as in [7] that every irreducible representation of $F_{\epsilon}^{\varphi}$ restricting to the counit of $F_{0}$ is one dimensional [3], and so the conditions in (2.9) hold for spec $F_{\epsilon}^{\varphi} \rightarrow \operatorname{spec} F_{0}$. Under certain additional restrictions on $\ell$, this result follows from [5], where dimensions of the irreducible representations are also calculated.

(iii) In contrast, it is a fundamental property of the central Hopf algebra embeddings featured in the representation theory, at roots of unity, of quantized enveloping algebras of semisimple Lie algebras (see, e.g., [8, Chapter 5; 13, Chapter 35]) that the conditions in (2.9) are not satisfied.

(iv) Letting $k$ be an arbitrary field, there is a $k$-bialgebra embedding of the classical coordinate ring $k\left[M_{n}\right]$ of $n \times n$ matrices into the center of the quantum coordinate ring $k_{q}\left[M_{n}\right]$ of $n \times n$ matrices, where $q$ is a primitive $t$ th root of unity in $k$ and $t$ is odd (cf. [17, Chapter 7]). Letting $X$ denote the group of convolution invertible characters of $k_{q}\left[M_{n}\right]$ that restrict to the counit on $k\left[M_{n}\right]$, there is an action of $X \times X$ on $k_{q}\left[M_{n}\right]$, by right and left winding automorphisms, that fixes $k\left[M_{n}\right]$ pointwise. It is shown in $[12,2.12]$ that the fibers of spec $k_{q}\left[M_{n}\right] \rightarrow \operatorname{spec} k\left[M_{n}\right]$ coincide with the $X \times X$ orbits in spec $k_{q}\left[M_{n}\right]$.

\section{REFERENCES}

1. E. Abe, Hopf algebras, Cambridge University Press, Cambridge, 1977.

2. M. Artin, W. Schelter, and J. Tate, Quantum deformations of $G L_{n}$, Comm. Pure Appl. Math. 44 (1991), 879-895.

3. M. Costantini, Personal communication, January, 1996.

4. M. Costantini and M. Varagnolo, Multiparameter Quantum Function Algebra at Roots of 1, Math. Ann. 306 (1996), 759-780.

5. _— A family of Azumaya algebras arising from quantum groups, C. R. Acad. Sci. Paris, Série I (1996), 127-132.

6. C. De Concini, V. Kac, and C. Procesi, Quantum Coadjoint Action, J. Amer. Math. Soc. 5 (1992), 151-189.

7. C. De Concini and V. Lyubashenko, Quantum Function Algebra at Roots of 1, Adv. Math. 108 (1994), 205-262.

8. C. De Concini and C. Procesi, Quantum Schubert cells and representations at roots of 1, Algebraic groups and Lie groups, a volume of papers in honour of the late R. W. Richardson (G. I. Lehrer, ed.), Australian Mathematical Society Lecture Series, no. 9, Cambridge University Press, Cambridge, 1997, pp. 127-160. 
9. K. R. Goodearl and R. B. Warfield, Jr., An introduction to noncommutative noetherian rings, London Mathematical Society Student Texts, vol. 16, Cambridge University Press, Cambridge, 1989.

10. A. Joseph, Quantum groups and their primitive ideals, Springer, New York, 1995.

11. E. S. Letzter, Primitive ideals in finite extensions of noetherian rings, J. London Math. Soc. (2) 39 (1989), 427-435.

12. On the quantum Frobenius map for general linear groups, J. Algebra 179 (1996), 115-126.

13. G. Lusztig, Introduction to quantum groups, Birkhäuser, Boston, 1993.

14. J. C. McConnell and J. C. Robson, Noncommutative noetherian rings, Wiley-Interscience, New York, 1987.

15. S. Montgomery, Hopf algebras and their actions on rings, Conference Board of the Mathematical Sciences, Regional Conference Series in Mathematics, Number 82, American Mathematical Society, Providence, 1993.

16. S. Montgomery and H.-J. Schnieder, Prime ideals in Hopf Galois extensions (to appear).

17. B. Parshall and J.-p. Wang, Quantum linear groups, Mem. Amer. Math. Soc. 89 (1991).

18. N. Reshetikhin, Multiparameter Quantum Groups and Twisted Quasitriangular Hopf Algebras, Letters Math. Phys. 20 (1990), 331-335.

19. J. C. Robson, Prime ideals in intermediate extensions, Proc. London Math. Soc. (3) 44 (1982), 372-384.

20. R. B. Warfield, Jr., Noetherian ring extensions with trace conditions, Trans. Amer. Math. Soc. 331 (1992), 449-463.

Department of Mathematics, Texas A\&M University, College Station, TX 77843

E-mail address: letzter@math.tamu.edu 